\title{
Neutron scattering study on the structure and dynamics of oriented lamellar phase microemulsions
}

\author{
Mihaela Mihailescu, Michael Monkenbusch,* Jürgen Allgaier, Henrich Frielinghaus, and Dieter Richter \\ Institut für Festkörperforschung, Forschungszentrum Jülich, D-52425 Jülich, Germany \\ Britta Jakobs and Thomas Sottmann \\ Institut für Physikalische Chemie, Universität zu Köln, Luxemburger Strasse 116, D-50939 Köln, Germany
}

(Received 29 May 2002; published 30 October 2002)

\begin{abstract}
Lamellar phases consisting of water and oil with intervening surfactant monolayer interfaces (eventually containing low molecular weight amphiphilic block-copolymers) are studied. Structural and dynamical investigations of oriented lamellar phases at the length scale of the intermembrane distance and beyond are performed using small-angle neutrons scattering and neutron spin-echo spectroscopy. The data analysis in terms of static and dynamic structure factors for a stack of elastic interfacial membranes yields information of the membrane curvature elasticity and membrane interactions in terms of phenomenological parameters such as the bending elastic modulus $\kappa$ and compression modulus $\bar{B}$, and on the dissipation related to the viscosity $\eta$. The influence of the block-copolymers anchored to the surfactant monolayers is studied for a series of lamellar phase microemulsions containing equal volume fraction of surfactant and polymer, by varying only the polymer molecular weight. Based on numerical evaluations and fits to the data the conditions of applicability of the available theoretical concepts are discussed.
\end{abstract}

DOI: 10.1103/PhysRevE.66.041504

PACS number(s): 83.80.Qr, 82.70.-y, 87.16.Dg, 61.12.-q

\section{INTRODUCTION}

Long-range ordered lamellar phases consisting of an alternation of water and oil layers separated by surfactant monolayers are one of the various topologically distinct structures which can self-organize in a ternary aqueous-oilsurfactant system, see Fig. 1. Obviously, for equal amounts of water and oil, the layers will have equal thicknesses and the mean distance between any two neighboring surfactant monolayers, denoted by $d_{f}$ (film-film distance), will be constant. This represents half the periodicity of the structure, generally denoted by $d$. The existence and stability of the lamellar phase in certain temperature-concentration windows of the phase diagram is determined, by a fine balance of forces, which act to prevent the melting of the ordered membrane stack into structures of higher entropy such as bicontinuous or droplets. For very flexible membranes (bending modulus $\kappa \simeq k_{B} T$ ), like those studied in this work, the large out-of-plane thermal fluctuations induce collisions between neighboring membranes. They give rise to a repulsive interaction which is caused by the reduction of entropy of the membrane fluctuations and is known under the name of Helfrich steric repulsion [1]. The Helfrich repulsion is of longrange nature $\left(\sim 1 / d_{f}^{2}\right)$ and competes with the Coulomb interaction (for electrically charged membranes) or with van der Waals attraction. For large distances the van der Waals attraction is very weak $\left(\sim 1 / d_{f}^{4}\right)$ in comparison with the steric repulsion. In the experimental work of Safinya et al. [2] on charge-neutral lamellar microemulsions of sodium dodecyl sulfate (SDS)/pentanol/water/dodecane, it was shown that for intermembrane distances $3.8 \mathrm{~nm}<d<16.4 \mathrm{~nm}$ the Helfrich steric interaction is dominant.

\footnotetext{
*Electronic address: m.monkenbusch@fz-juelich.de
}

Many studies tried to vary the balance of the interaction forces by changing the amphiphilic layer composition, e.g., adding ionic surfactants to a basic nonionic system [3], adding long chain polymers to a surfactant system [4] or adding polymers which are grafted on the surfactant layers $[5,6]$. It was shown $[7,8]$, that anchoring polymers on the surfactant interface has dramatic influences on the extension of the bicontinuous single-phase region in the phase diagram. The position and boundaries of a lamellar phase appearing in a basic surfactant system are also modified. Long polymers act on the phase diagram as to expand the random, bicontinuous phase domain, while suppressing the formation of the lamellar phase [7], possibly due to confinement effects as reported in Ref. [4]. Recently reported SANS results [8] were interpreted in the light of Ginzburg-Landau and Gaussianrandom-field theories. Tethered polymers on surfactant layers modify the elastic moduli $\kappa$ and $\bar{\kappa}$. The added polymers have profound effect on the correlation lengths of the structure and on the phase diagram. The observations indicated that

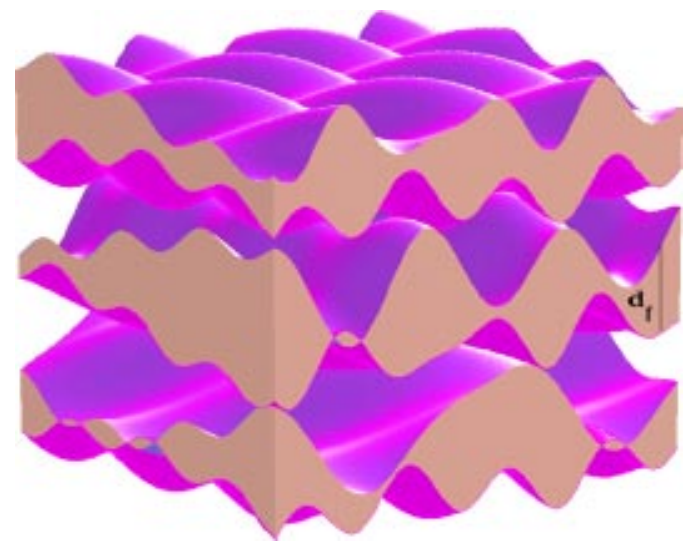

FIG. 1. Schematic picture of a liquid lamellar structure consisting of oil/surfactant/water layers (computer animation). 
long polymers favor saddle-splay deformations that are responsible for the suppression of the lamellar phase. Interestingly, however, upon addition of polymers, the appearance of lamellar islands, at higher dilution than normally expected for the pure $\mathrm{C}_{10} \mathrm{E}_{4} /$ water/decane system has been observed. This stimulated the experimental work on oriented lamellar phases containing block copolymers. Due to their symmetry such investigations allow for a more straightforward description and determination of the characteristic parameters such as structural size or elastic properties.

\section{STRUCTURE FACTORS}

\section{A. The static structure factor of a membrane stack}

In harmonic approximation the Hamiltonian describing a stack of membranes (only terms up to the second order of the undulation amplitude and its derivatives are considered) can be written [1] as

$$
H=\frac{1}{2} \bar{B}\left(\frac{\partial u}{\partial z}\right)^{2}+\frac{1}{2} \kappa_{c}\left(\frac{\partial^{2} u}{\partial x^{2}}+\frac{\partial^{2} u}{\partial y^{2}}\right)^{2},
$$

where $\bar{B}$ is the compression modulus at constant chemical potential, and $\kappa_{c}$ relates to the single-membrane bending modulus $\kappa$ by $\kappa_{c}=\kappa / d_{f}$. The compression modulus $\bar{B}$ is related to the derivative of the membrane interaction energy density. The way in which it varies with the separation distance $d_{f}$ is determined by the type of interaction that exists between membranes. If undulation forces dominate, then $\bar{B}$ $\propto d_{f} /\left(d_{f}-a\right)^{4}$, where $a$ is the membrane thickness. From Eq. (1) and the energy equipartition theory, the Fourier components of the undulation amplitude are [9]

$$
\left\langle\left|U_{k}\right|^{2}\right\rangle=\frac{k_{B} T}{\bar{B} k_{z}^{2}+\kappa_{c} k_{\perp}^{4}},
$$

where \langle\rangle represent the average over the ensemble of membranes. The intensity scattered by the stack of membrane was calculated by Caillé [10]:

$$
\begin{aligned}
I(q) & =\left|f\left(q_{z}\right)\right|^{2} \sum_{n} \int d^{2} \vec{\rho} e^{i q_{z} n d} e^{i q_{\perp}} \vec{\rho}\left\langle e^{i q_{z}\left(u_{n}(\rho)-u_{0}(0)\right.}\right\rangle \\
& =\left|f\left(q_{z}\right)\right|^{2} S(q),
\end{aligned}
$$

where $q_{z}$ and $q_{\perp}$ describe the components of the scattering wave vector parallel and, respectively, perpendicular to the normal vector to the lamellar plane. Correspondingly, $z$ $=n d_{f}$ and $\vec{\rho}$ are the components of the real-space position vector $\overrightarrow{\mathrm{r}}=\left(\rho_{x}, \rho_{y}, z\right)$. The factors $\left|f\left(q_{z}\right)\right|^{2}$ and $S(q)$ denote the form factor of a membrane and the membrane stack structure factor, respectively. In the harmonic approximation any combination of the variable $u_{n}(\rho)$, which describes the displacement of the membrane from an average plane, follows a Gaussian distribution, which implies

$$
\left\langle e^{i q_{z}\left(u_{n}(\rho)-u_{0}(0)\right.}\right\rangle=e^{-1 / 2 q_{z}^{2}\left\langle\left|u_{n}(\rho)-u_{0}(0)\right|^{2}\right\rangle}
$$

$\left\langle\left|u_{n}(\rho)-u_{0}(0)\right|^{2}\right\rangle$ is related to the Fourier transformed undulation amplitudes by

$$
\left\langle\left|u_{n}(\rho)-u_{0}(0)\right|^{2}\right\rangle=\frac{2}{(2 \pi)^{3}} \int d^{3} \overrightarrow{\mathrm{k}}\left(1-e^{-i \overrightarrow{\mathrm{kr}}}\right)\left\langle\left|U_{\overrightarrow{\mathrm{k}}}\right|^{2}\right\rangle .
$$

Inserting Eqs. (2), (4), and (5) in Eq. (3) yields finally (see the Appendix for the derivation scheme)

$$
S(q) \propto \sum_{n=-\infty}^{n=+\infty} e^{i q_{z} n d} I_{\rho}\left(n ; q_{z}, q_{\perp}\right),
$$

where

$$
\begin{aligned}
I_{\rho}= & \int d \rho \rho J_{0}\left(q_{\perp} \rho\right) \\
& \times \exp [-k_{B} T /(2 \pi)^{3} q_{z}^{2} \overbrace{\int d^{3} \overrightarrow{\mathrm{k}}\left(1-e^{-\overrightarrow{\mathrm{k} r}}\right)\left\langle\left|U_{\vec{k}}\right|^{2}\right\rangle}^{I_{\overrightarrow{\mathrm{k}}}}] .
\end{aligned}
$$

With $\left\langle\left|U_{\vec{k}}\right|^{2}\right\rangle$ given by Eq. (2) and $\overrightarrow{\mathrm{r}}=\left(\rho_{x}, \rho_{y}, z=n d\right)$, the integral over the mode wave vector $\vec{k}$ can be further evaluated to

$$
I_{\overrightarrow{\mathrm{k}}}=\frac{2 \pi^{2}}{\bar{B} \sqrt{\alpha}} \int_{0}^{k_{\max }} d k_{\perp} \frac{1}{k_{\perp}}\left[1-J_{0}\left(k_{\perp} \rho\right) e^{-\sqrt{\alpha} k_{\perp}^{2}|z|}\right]
$$

with $\alpha=\kappa_{c} / \bar{B}$, and $J_{0}$-the Bessel function of order 0 . The final result, as extended in the Appendix, is written as

$$
\begin{aligned}
I_{\rho}\left(n ; q_{z}, q_{\perp}\right)= & e^{-\mu / 2\left[2 \gamma+E_{1}\left(k_{\max }^{2} n d \sqrt{\alpha}\right)\right]} \frac{1}{q_{\perp}^{2}}\left(\frac{k_{\max }}{2 q_{\perp}}\right)^{-\mu} \\
& \times \int_{0}^{\infty} d x J_{0}(x) x^{1-\mu} e^{-\mu / 2 E_{1}\left(x^{2} /\left(4 \sqrt{\alpha} q_{\perp}^{2} n d\right)\right)}
\end{aligned}
$$

with $\mu=q_{z}^{2} /(4 \pi) k_{B} T /(\bar{B} \sqrt{\alpha}), x=q_{\perp} \rho$ and $E_{1}$ the exponential integral function of order 1 . If thermal undulations would not be present, i.e., $\mid\left\langle\left. U_{\overrightarrow{\mathrm{k}}}\right|^{2}\right\rangle=0$, the scattered intensity would consist of a series of $\delta$-functions (Bragg peaks) situated at $q_{\mathrm{mf}}=m 2 \pi / d_{f}$. The undulations yield considerable off-peak intensity and convert the $\delta$ peaks to power-law singularities and suppress higher-order peaks.

The expression (6) was used in the fitting procedure of the SANS experimental data as outlined in Sec. IV A. The form factor of the surfactant monolayer $\left|f\left(q_{z}\right)\right|^{2}$ mainly affects the intensity in the high- $q$ part, $(q>1 / a)$, where the intramembrane correlation effects contribute to the scattered intensity [ $a \simeq 1.2 \mathrm{~nm}$, according to Ref. [8]]. In the region of the firstorder scattering peaks where the fits were carried out, the form factor is almost a constant. The Caille theory [10] considers an infinite number of lamellae in the stack. In reality, the extension of the stack is finite, however large. In order to avoid cut-off oscillations in the result, an effective finite size 
effect was accounted for by multiplying the quantity under the sum with a Gaussian $\exp \left[-1 / 2\left(n d / L_{z}\right)^{2}\right]$, where $L_{z}$ covers a few hundreds nanometers along the normal to the lamellar planes. The summation is then carried out up to an $n_{\max }$ sufficiently large to assure the convergence of the thus modified sum. This limitation has the effect of rounding off the divergence of the intensity at the peak position $q=q_{\mathrm{mf}}$ however, to a much lower extent than the instrumental resolution but to an extent that a numerical resolution convolution becomes feasible. In order to compare the experimental data with the theoretical scattering intensity [Eq. (6)] the latter needs to be convoluted with the three-dimensional resolution function of the SANS instrument. The measured intensity will then be compared to

$$
I_{M}\left(\overrightarrow{\mathrm{q}}_{0}\right)=\int d^{3} \overrightarrow{\mathrm{q}} \mathcal{R}\left(\overrightarrow{\mathrm{q}}-\overrightarrow{\mathrm{q}}_{0}\right) I(\overrightarrow{\mathrm{q}})
$$

According to Ref. [11] for the resolution function $\mathcal{R}(\overrightarrow{\mathrm{q}})$ a Gaussian ellipsoid was assumed.

\section{B. The dynamic structure factor of a membrane stack}

At low wave vectors, around or below the first correlation peak $\left(q \leqslant q_{1 \mathrm{f}}\right)$ is the realm of the hydrodynamic regime. There interactions between different membranes yield a richer and more complicated relaxation mode structure which arises from repulsive and/or attractive and hydrodynamic forces between layers and from coupling between concentration and layer displacement modes. The hydrodynamic model for a lipid-water lamellar phase was originally formulated by Brochard and Lennon [12] and was further developed by e.g., Nallet et al. [13], Ramaswamy [14], Schilling et al. [15].

At higher $q\left(q>q_{1 \mathrm{f}}\right)$, the motions probed are no longer collective, and concentration fluctuations now arise from the surfactant layer displacement with respect to the surrounding solvent. In the work of Schilling et al. [15], the hydrodynamic model has been extended to describe ternary oilsurfactant-water system in the asymptotic limit $\left(q d_{f} \gg 1\right)$ which is relevant for NSE experiments. All hydrodynamic models show that the relaxation rates for all modes behave asymptotically for large $k$ as

$$
\omega^{\infty}(k)=k^{3} \kappa /\left[2\left(\eta_{\text {oil }}+\eta_{\text {water }}\right)\right] .
$$

This short wavelength limit is equivalent to the dispersion relation of a single-membrane undulation mode (membrane Zimm dynamics) $[14,16]$ in a solvent of viscosity given by the average between the water and oil viscosities. Thus, in the high- $q$ (local) regime, the theories predict the membrane Zimm dynamics, as used by Zilman and Granek [17] for the bicontinuous phase.

In order to describe the NSE data $S(q, t) / S(q)$, a dynamical version of the Caille theory [10] using the dispersion relation expressed in Eq. (11) can also be conceived. Then in Eq. (5) the displacement correlator $\left\langle u_{k}(0) u_{-k}(t)\right\rangle$ changes from $e^{i \overrightarrow{\mathrm{kr}}}$ at $t=0$ to $e^{i \overrightarrow{\mathrm{kr}}} e^{-\omega\left(k_{\perp}\right) t}$. This leads to the timedependent expression for $I_{\overrightarrow{\mathrm{k}}}$ :

$$
I_{\overrightarrow{\mathrm{k}}, t}=\frac{2 \pi^{2}}{\bar{B} \sqrt{\alpha}} \int_{0}^{k_{\max }} \frac{d k_{\perp}}{k_{\perp}}\left[1-J_{0}\left(k_{\perp} \rho\right) e^{-\sqrt{\alpha} k_{\perp}^{2}|z|} e^{-\omega\left(k_{\perp}\right) t}\right] .
$$

In general, we need to distinguish between the mode wave vector $\vec{k}$ and the momentum transfer $\vec{q}$ of the scattering experiment. From Eq. (6) it is evident that at a given $q$ many modes contribute to the observed relaxation, i.e., the experimental wave vector $q$ and the mode wave vector $k$, respectively, $k_{\perp}$ must strictly be distiguished.

The cutoff in the small wavelength limit $k_{\max } \simeq \pi / a$ is required to achieve the convergence of the integral and is determined by the smallest undulation-mode wavelength, in the order of the molecular size.

A numerical evaluation of Eq. (12) is only feasible for simple expressions for the dispersion $\omega(\overrightarrow{\mathrm{k}})$, here we use the asymptotic expression Eq. (11), i.e., $\omega(\overrightarrow{\mathrm{k}}) \propto k_{\perp}^{3}$. The latter simplification also requires a lower cutoff $k_{\min }$ for the integration in Eq. (12), see below.

\section{The asymptotic limit $\left(q \gg q_{1 f}\right)$}

In the asymptotic limit the local fluctuations of a singlemembrane patch are probed. Taking $n=0$ the expression for the structure factor of a single membrane can be easily derived from Eq. (3)

$$
S(\overrightarrow{\mathrm{q}}) \propto \int d^{2} \vec{\rho} e^{i q_{\perp} \vec{\rho}}\left\langle e^{i q_{z}(u(\rho)-u(0))}\right\rangle .
$$

In this case the intermembrane correlation can be disregarded $\left(\vec{k}=\vec{k}_{\perp}\right)$ and the Fourier components of the undulation amplitude become

$$
\left\langle\left|U_{\overrightarrow{\mathrm{k}}}\right|^{2}\right\rangle=\left\langle U_{\overrightarrow{\mathrm{k}}}^{\overrightarrow{\mathrm{k}}} U_{-\overrightarrow{\mathrm{k}}}\right\rangle=\frac{k_{\mathrm{B}} T}{\kappa k^{4}},
$$

where the in-plane wave vector $\overrightarrow{\mathrm{k}}$ labels the undulation modes. Expression (14) takes account for the membrane bending only; any interaction effects are ignored. The resulting scattering function can be written

$$
\begin{aligned}
S(\overrightarrow{\mathrm{q}}) \propto & \int_{0}^{\rho_{\max }} d \rho \rho \mathrm{J}_{0}\left(q_{\perp} \rho\right) \\
& \times \exp \left[-\frac{q_{z}^{2}}{(2 \pi)^{2}} \int d^{2} \overrightarrow{\mathrm{k}}\left(1-e^{-i \overrightarrow{\mathrm{k}} \rho}\right)\left\langle\left|U_{\overrightarrow{\mathrm{k}}}\right|^{2}\right\rangle\right] .
\end{aligned}
$$

In correspondence to Eq. (12) above, the expression for the single-membrane dynamic structure factor reduces to

$$
\begin{aligned}
S(\overrightarrow{\mathrm{q}}, t) \propto & \int_{0}^{\rho_{\max }} d \rho \rho \mathrm{J}_{0}\left(q_{\perp} \rho\right) \exp \left\{-\frac{k_{\mathrm{B}} T}{2 \pi \kappa} q^{2} \mu^{2}\right. \\
& \left.\times \int_{k_{\min }}^{k_{\max }} \frac{d k}{k^{3}}\left[1-\mathrm{J}_{0}(k \rho) e^{-\omega^{\infty}(k) t}\right]\right\}
\end{aligned}
$$


TABLE I. The compositions of the investigated lamellar phase microemulsions, $\sigma_{R}=\sigma\left(R_{\mathrm{PEP}}^{2}+R_{\mathrm{PEO}}^{2}\right)$.

\begin{tabular}{ccccccc}
\hline \hline Surfactant & $\gamma$ & $\delta$ & $\phi_{s}$ & $\phi_{\delta}$ & $\sigma\left[\mathrm{nm}^{-2}\right]$ & $\sigma_{R}$ \\
\hline $\mathrm{C}_{10} \mathrm{E}_{4}$, no polymer & 0.305 & 0 & 0.270 & 0 & 0 & 0 \\
$\mathrm{C}_{10} \mathrm{E}_{4}+\mathrm{PEP}_{1} / \mathrm{PEO}_{1}$ & 0.172 & 0.123 & 0.151 & 0.123 & 0.0373 & 0.868 \\
$\mathrm{C}_{10} \mathrm{E}_{4}+\mathrm{PEP}_{2} / \mathrm{PEO}_{2}$ & 0.170 & 0.121 & 0.149 & 0.121 & 0.0209 & 0.888 \\
$\mathrm{C}_{10} \mathrm{E}_{4}+\mathrm{PEP}_{5} / \mathrm{PEO}_{5}$ & 0.171 & 0.118 & 0.151 & 0.118 & 0.0090 & 0.918 \\
\hline \hline
\end{tabular}

with $\omega^{\infty}(k)=k^{3} \kappa / 4 \bar{\eta}, \bar{\eta}$ denotes the average viscosity of water and oil. The above scattering function has been derived by Zilman and Granek [17] for the asymptotic, high- $q$ regime dynamics, and was proven to give a good description of the data if a full numerical evaluations of Eq. (16) with the appropriate integration limits is performed [18]. For the lamellar phase integration limits $k_{\min }=\epsilon \pi / d_{f}$ and, $r_{\max }$ $\simeq d_{f} / \epsilon$, in reciprocal and real space respectively, are expected to be determined by a correlation length of the order of the intermembrane distance $d_{f}$, with a not exactly known factor $\epsilon \simeq 1$. In the limit of large bending rigidities $(\kappa$ $\gg k_{B} T$ ), Eq. (16) reduces to a simple stretched exponential function:

$$
S(q, t) \simeq S(q) e^{-\left(\Gamma_{q} t\right)^{\beta}}
$$

with and $\beta=2 / 3$, and $\Gamma_{q} \propto \sqrt{k_{B} T / \kappa}\left(k_{B} T / \eta\right) q^{3}$ denoting the relaxation rate [17].

\section{THE INVESTIGATED MICROEMULSIONS IN LAMELLAR PHASE}

\section{A. Compositions}

For the neutron scattering investigations of the lamellar phase, a series of microemulsions were prepared based on the nonionic surfactant decyl-polyglycol-ether $\left(\mathrm{C}_{10} \mathrm{E}_{4}\right)$ emulsifying equal amounts of water and decane. Three of the samples contained additionally equal volume fractions of amphiphilic block-copolymers $\mathrm{PEP}_{x}-\mathrm{PEO}_{y}$ of different molecular weights, where $x, y$ denote the molecular weights of the two blocks in $\mathrm{kg} / \mathrm{mol}$. By using hydrogeneous surfactants and polymers in combination with deuterated solvents ( $h$ surfactant $/ \mathrm{D}_{2} \mathrm{O} / d$ decane) the samples were studied under conditions of film contrast. There due to the large difference in the scattering length between $\mathrm{H}$ and $\mathrm{D}$, the relevant neutron scattering intensity is provided by the surfactant film. The compositions of the samples are summarized in Table I.

These were chosen such as to obtain lamellar single phases at not too high surfactant concentration and to preserve as much as possible the membrane volume fraction. For the pure $\mathrm{C}_{10} \mathrm{E}_{4}$ system, higher surfactant concentrations are required in order to obtain the lamellar structure. Preserving also the polymer volume fractions relative to the surfactant, the number density of polymer anchored to the membrane $(\sigma)$ decreases with increasing molecular weight. As seen in Table I, the product $\sigma\left(R_{\mathrm{PEP}}^{2}+R_{\mathrm{PEO}}^{2}\right)$ is nearly constant. The symbols $\gamma$ and $\delta$ are used to denote the mass fractions of surfactant + polymer in solution, and of polymer in surfactant + polymer, respectively.
The surfactant volume fractions $\phi_{s}$ refer to the $\mathrm{C}_{10} \mathrm{E}_{4}$ only, and are calculated considering the $1.7 \mathrm{wt} \%$ solubility of $\mathrm{C}_{10} \mathrm{E}_{4}$ in $d$ decane [19]. The samples with polymers were prepared by adding to this basic decane $/ \mathrm{C}_{10} \mathrm{E}_{4} /$ water mixture equal amounts of polymers. $\phi_{\delta}$ denotes the volume fraction of polymer in the surfactant layer, i.e., in $\mathrm{C}_{10} \mathrm{E}_{4}+$ polymer mixture after subtraction of the $\mathrm{C}_{10} \mathrm{E}_{4}$ in solution.

\section{B. Polymers}

The block copolymers were synthesized by anionic polymerization at FZ-Jülich (IFF) by Allgaier et al. [20]. They have similar chemical structure as the surfactant itself. The total molecular weights and the end-to-end distances of each block in its solvent are presented in Table II.

\section{Phase diagram}

The lamellar phase of the nonionic $\mathrm{C}_{10} \mathrm{E}_{4}$ surfactant in symmetric water-oil mixtures without any co-surfactant can only be achieved at surfactant mass fractions beyond $\gamma$ $=0.23$ (see the phase diagram presented in Fig. 2) [38]. The addition of an amphiphilic block-copolymers of not too high molecular weight leads to the appearance of a one-phase lamellar island at not too high surfactant concentrations, i.e., at $\gamma \simeq 0.13$ (Fig. 2). All three systems containing polymer could be obtained at the same membrane volume fraction. The presented cross-section through the phase diagram displays the domain of the one-phase (1 $\Phi)$ bicontinuous microemulsions embedding the lamellar island, corresponding to the pure $\mathrm{C}_{10} \mathrm{E}_{4}$ system and also to a mass fraction $\delta$ $=0.118$ of $\mathrm{PEP}_{5}-\mathrm{PEO}_{5}$ in surfactant. The arrows indicate the points in the phase diagram were the lamellar phases for the neutron scattering experiments were prepared. A lamellar phase for longer polymers, e.g., $\mathrm{PEP}_{10}-\mathrm{PEO}_{10}$, was not observed in this concentration range. While scanning the temperature the phase boundaries were established by visual inspection of the samples immersed in a water bath. When viewed through crossed polarizers the formation of the large domains of lamellar stacks is noticed by the birefringence displayed. The temperature scale of the phase diagram

TABLE II. Polymers used in the compositions of the investigated lamellar phase microemulsions.

\begin{tabular}{cccc}
\hline \hline Polymer & Molecular weight $[\mathrm{g} / \mathrm{mol}]$ & $R_{\mathrm{PEO}}[\mathrm{nm}]$ & $R_{\mathrm{PEP}}[\mathrm{nm}]$ \\
\hline $\mathrm{PEP}_{1} / \mathrm{PEO}_{1}$ & 2700 & 3.49 & 3.33 \\
$\mathrm{PEP}_{2} / \mathrm{PEO}_{2}$ & 4730 & 4.85 & 4.36 \\
$\mathrm{PEP}_{5} / \mathrm{PEO}_{5}$ & 10500 & 7.71 & 6.69 \\
\hline \hline
\end{tabular}




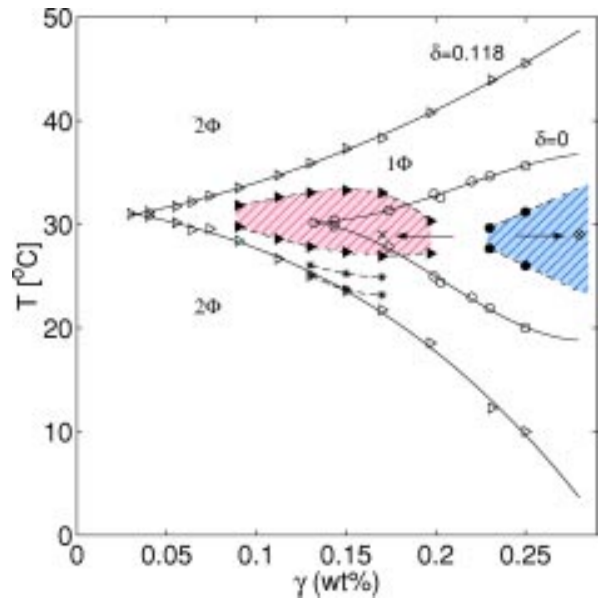

FIG. 2. Part of the phase diagram of a microemulsion $\mathrm{C}_{10} \mathrm{E}_{4} / \mathrm{H}_{2} \mathrm{O} / h$ decane without polymer (circles), or containing 11.8 wt $\% \mathrm{PEP}_{5}-\mathrm{PEO}_{5}$ in surfactant (triangles). The corresponding lamellar phase islands are indicated by the shaded regions. The arrows indicate the respective positions in the phase diagram corresponding to the investigated systems: $(X)$ with polymers, $(\otimes)$ without polymer. At the lowest phase boundary the appearance of an ordered, highly viscous phase was observed $(\star)$.

shown in Fig. 2 corresponds to hydrogeneous compounds. The actual investigated samples were prepared in film contrast, containing deuterated water and decane. This shifts the phase boundaries to slightly lower temperatures whereas the tips of the one-phase domains remains at almost the same composition. For the systems containing $\mathrm{PEP}_{1}-\mathrm{PEO}_{1}$ and $\mathrm{PEP}_{2}-\mathrm{PEO}_{2}$, it was verified that the lamellar phases also exist at $\gamma=0.17$ in approximately the same temperature window which, however, slightly narrows with decreasing polymer size.

\section{NEUTRON SCATTERING EXPERIMENTS}

In order to orient the lamellar domains, the samples were filled into specially designed sample cells [21]. A cell consists mainly of a copper block enframing a stack of very thin lamellar quartz plates $2.5 \mathrm{~mm}$ wide, $50 \mathrm{~mm}$ long, with a stack pace of $0.2 \mathrm{~mm}$, fixed in copper combs. Copper blocks in thermal contact with the sample cell were used to control the sample temperature. All the samples were studied at $26^{\circ} \mathrm{C}$, assuring a safe distance from the boundaries to the bicontinuous phase. Using an water bath the solutions were initially brought to a temperature somewhat higher than $26^{\circ} \mathrm{C}$, (not leaving the lamellar domain) and then, with a syringe, immediately injected into the sample cells. The orientation is realized during injection by a combination of shear flow and the close proximity of the quartz plates.

The lamellar phases were investigated using the smallangle neutrons scattering (SANS) (KWS-2) and neutron spin-echo spectroscopy (NSE) instruments at FRJ2 in Jülich. Due to very strong scattering, only short SANS counting times, of $10 \mathrm{~min}$ for the systems with polymer and $1 \mathrm{~h}$ for the pure surfactant system were needed. The background turned out to be insignificant and no subtraction was performed. The time interval between the SANS and the NSE
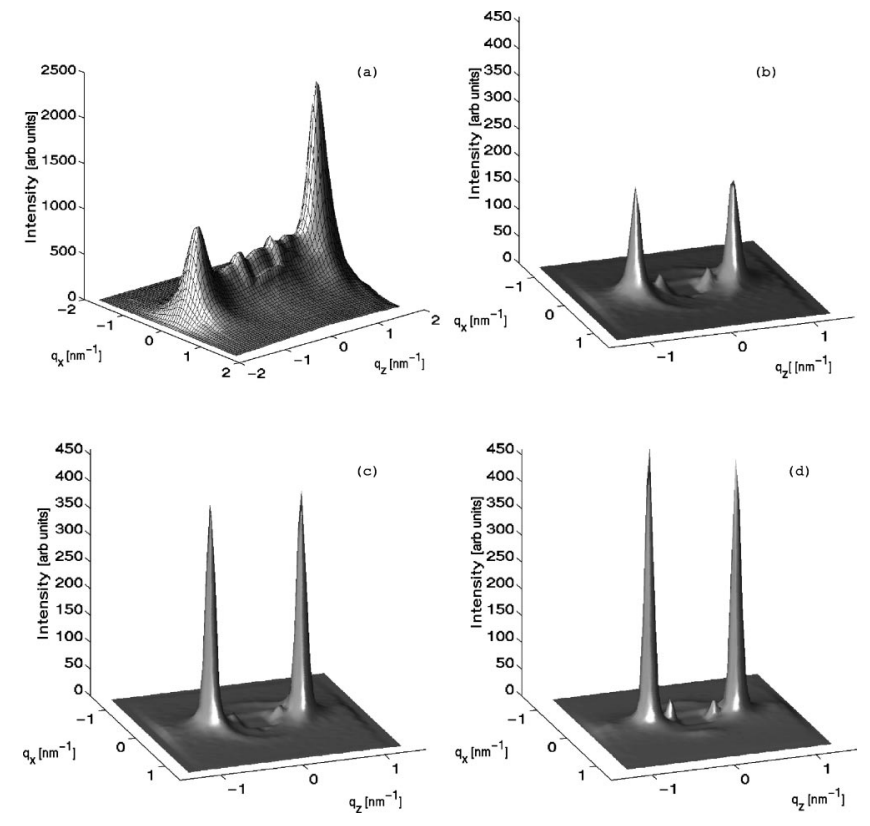

FIG. 3. Scattering intensity from oriented lamellar phases corresponding to: (a) basic $\mathrm{C}_{10} \mathrm{E}_{4}$ system, (b) $\mathrm{PEP}_{1}-\mathrm{PEO}_{1}$, (c) $\mathrm{PEP}_{2}-\mathrm{PEO}_{2}$, and $(d) \mathrm{PEP}_{5}-\mathrm{PEO}_{5}$.

experiments was in general a few hours. During this time the sample temperature was maintained constant.

\section{A. Small angle scattering results}

Preliminary SANS investigations of the oriented lamellar phases allowed for an assessment of the degree of orientation and the determination of the scattering peak position in each case. The experiments have been performed at the KWS-2 SANS camera in the cold neutron guide hall at the FRJ2reactor in Jülich. The neutron wavelength used was $\lambda$ $=0.7 \mathrm{~nm}$ and the detector distance was $1.2 \mathrm{~m}$. Each pixel position on the $2 D$-detector correspond to a certain $\vec{q}$ $\equiv\left(q_{x}, q_{y}, q_{z}\right) \equiv\left(\overrightarrow{\mathrm{q}}_{\perp}, q_{z}\right)_{\text {sample }}$ on the surface of an Ewald sphere of large radius, i.e., nearly planar in the detected field. The sample was oriented in the beam such that the lamellar planes were parallel to the beam direction. In the presentations the $q_{z}$ axis of the instrument is normal to the lamellar planes. Therefore, the maxima of intensity are aligned along $q_{z}$. The maps of the scattering intensities are shown in Fig. 3 $(a-d)$. They display results from the basic system and the three polymer containing systems: $\mathrm{PEP}_{1}-\mathrm{PEO}_{1}, \mathrm{PEP}_{2}-\mathrm{PEO}_{2}$, and $\mathrm{PEP}_{5}-\mathrm{PEO}_{5}$, respectively. As can be inferred from the small radial spread of the reflection peaks and the very weak isotropic intensity ring a good orientation has been obtained. In all cases the orientation obtained for the systems containing polymer was better than for the basic system.

In order to determine the bending modulus $\kappa$ and the strength of the intermembrane interaction in terms of the compression modulus $\bar{B}$, the SANS intensity was analyzed by using the Caillé's treatment [10], Eq. (3). For this purpose an involved numerical procedure based on Eq. (A15) explained in the Appendix (in combination with a convolution with the experimental resolution) has been used to describe 


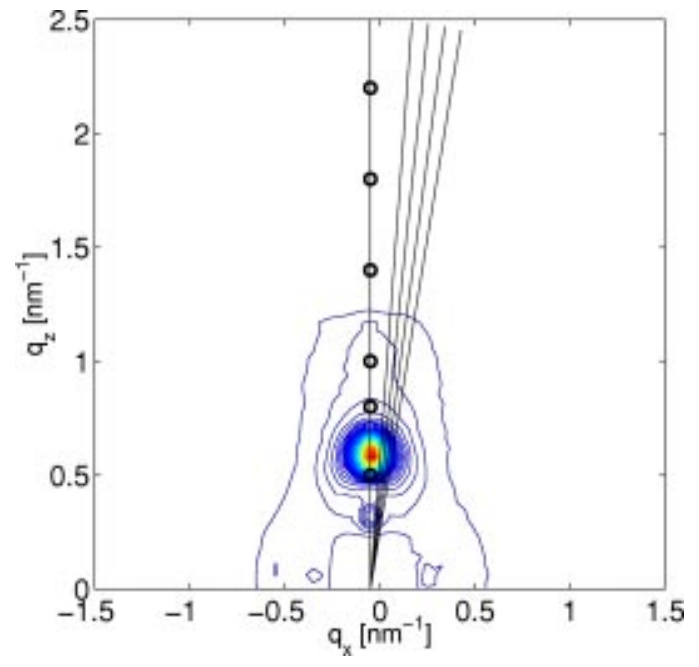

FIG. 4. Contour representation of the scattering intensity corresponding to the $\mathrm{PEP}_{5}-\mathrm{PEO}_{5}$ system. $\left(q_{x}, q_{z}\right)$ are the components on the detector plane corresponding to the direction parallel and, respectively, perpendicular to the membranes plane. The cuts at $5^{\circ}$, $7^{\circ}, 9^{\circ}, 11^{\circ}$ indicated by the cross-lines were used in the static structure factor analysis. The circles on the $0^{\circ}$ line indicate the $q$ positions where the dynamic structure factor $S(q, t)$ was measured by NSE.

the experimental data. Since a complete fit of the intensity map proved to need excessive computation time, a few cross cuts at different angles as indicated in Fig. 4 were used to be fitted simultaneously by the numerical procedure in each case [39]. The fitted range of data points was limited to the first-order scattering peak. The smaller peaks in the intensity maps, situated at $q \simeq q_{1 \mathrm{f}} / 2$, are attributed to a residual differences in the scattering length density of water and oil. $d$ decane and $\mathrm{D}_{2} \mathrm{O}$ are not perfectly matched yielding intensity at the periodicity of the chemical composition $q_{1 \mathrm{f}} / 2$ rather than at that $\left(q_{1 \mathrm{ff}}\right)$ of the membrane-membrane stack. Another contribution to the intensity at the same position would result from small asymmetries in the water/oil layer thicknesses. They are not included in the model.

The quality of the fits using this procedure can be judged from Fig. 5. (A fit for the basic $\mathrm{C}_{10} \mathrm{E}_{4}$ system could not be achieved with the current procedure. Numerical instabilities, especially at higher $q$ 's, where the correlation peak for this system is found prevented convergence. Despite the many approximation involved it can be seen that the simultaneous fits are describing the data very well. The values determined for the parameters $d_{f}, \kappa$, and $\bar{B}$ are presented in Table III.
TABLE III. Fit parameters of Caille formulation for the lamellar phase microemulsions, $\eta_{1}=q_{1 \mathrm{f}}^{2} \mathrm{f}\left[8 \pi \sqrt{\bar{B} \kappa_{c}}\right]$.

\begin{tabular}{ccccc}
\hline \hline Surfactant & $d_{f}[\mathrm{~nm}]$ & $\bar{B}\left[k_{B} T / \mathrm{nm}^{3}\right]$ & $\kappa\left[k_{B} T\right]$ & $\eta_{1}$ \\
\hline $\mathrm{C}_{10} \mathrm{E}_{4}+\mathrm{PEP}_{1} / \mathrm{PEO}_{1}$ & 10.57 & 0.1972 & 0.872 & 0.110 \\
$\mathrm{C}_{10} \mathrm{E}_{4}+\mathrm{PEP}_{2} / \mathrm{PEO}_{2}$ & 11.40 & 0.2138 & 0.759 & 0.101 \\
$\mathrm{C}_{10} \mathrm{E}_{4}+\mathrm{PEP}_{5} / \mathrm{PEO}_{5}$ & 12.04 & 0.2410 & 0.728 & 0.090 \\
\hline \hline
\end{tabular}

The determined interlayer distances increase with the polymer size. The first-order pseudo-Bragg peaks are positioned at $q_{1 \mathrm{f}}=0.52 \mathrm{~nm}^{-1}$ for $\mathrm{PEP}_{5}-\mathrm{PEO}_{5}, q_{1 \mathrm{f}}=0.55 \mathrm{~nm}^{-1}$ for $\mathrm{PEP}_{2}-\mathrm{PEO}_{2}$, and $q_{1 \mathrm{f}}=0.59 \mathrm{~nm}^{-1}$ for $\mathrm{PEP}_{1}-\mathrm{PEO}_{1}$. The observed shift cannot be related to surfactant concentration which remained constant for the three systems (Table I), but needs to be connected to differences in the length of the polymers anchored to the surfactant membranes. We discuss these matters in Sec. V.

The thus obtained bending modulus $\kappa$ slightly decreases with polymer length. This might be attributed to polymer surface density effects, since in our case the polymer density on the membrane is inversely proportional to their molecular size (at fixed polymer volume fraction). Performing the full numerical integration for the determination of $\kappa$, all undulation modes are accounted for in the evaluation. This suggests that the determined values of $\kappa$ correspond to the bare bending modulus of the membrane, as described also in Ref. [18].

Hiergeist and Lipowsky [22] found that, for a number density of polymer $\sigma$ in the membrane, the effective bare bending modulus of the layer is increased by an amount $\Delta \kappa=\left(k_{\mathrm{B}} T / 12\right)(1+\pi / 2) \sigma\left(R_{\mathrm{PEP}}^{2}+R_{\mathrm{PEO}}^{2}\right)$ compared to the pure membrane. Applying this expression with $\kappa_{0}$ $=0.92 k_{B} T$ to a pure $\mathrm{C}_{10} \mathrm{E}_{4}$ membrane [23], a value of $\kappa_{\text {eff }}$ $\simeq 1.11 k_{B} T$ results for all three polymer containing systems. The values obtained by the present analysis are slightly below those that result from the expression of Hiergeist and Lipowsky. The compression modulus $\bar{B}$ is of the order of $0.1 k_{B} T / \mathrm{nm}^{3}$, slightly increasing with the polymer length. Using the parameters from Table III a complete twodimensional intensity map was computed and compared to the experimental data. Figure 6 displays the scattering intensity comparison for the $\mathrm{PEP}_{5}-\mathrm{PEO}_{5}$ system. As derived in Refs. [10,37], Eq. (6) may lead to an algebraic divergence of the intensity at $q_{\mathrm{mf}}=m 2 \pi / d_{f}$, where the intensity along the two directions $q_{z}$ and $q_{\perp}$ follows an inverse power-law behavior,
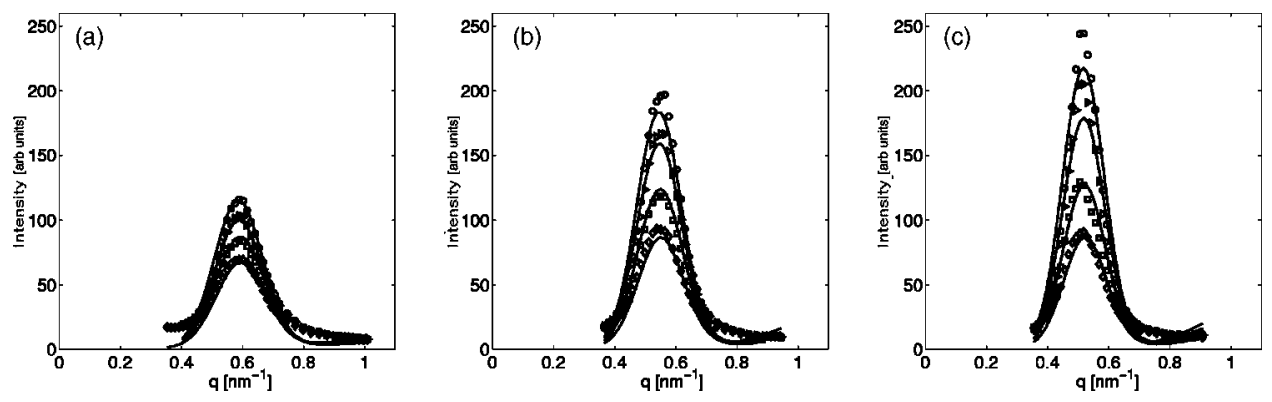

FIG. 5. Simultaneous fits using Eq. (A15) of cross sections at four different angles $\left(6^{\circ}, 7^{\circ}, 9^{\circ}\right.$, and $\left.11^{\circ}\right)$ through the SANS intensity distribution on the $\left(q_{x}, q_{z}\right)$ detector plane: (a) $\mathrm{PEP}_{1}-\mathrm{PEO}_{1}$, (b) $\mathrm{PEP}_{2}-\mathrm{PEO}_{2}$, and (c) $\mathrm{PEP}_{5}-\mathrm{PEO}_{5}$. 


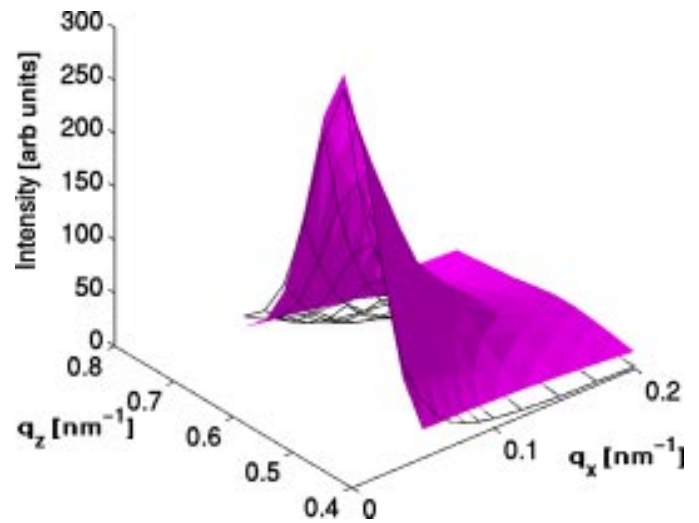

FIG. 6. Part of the intensity map reproduced with the Caille formalism (mesh), in comparison with the experimental intensity (solid surface) for the $\mathrm{PEP}_{5}-\mathrm{PEO}_{5}$ system.

$$
\begin{gathered}
S\left(q_{z} \simeq q_{\mathrm{mf}}, q_{\perp}=0\right) \propto \frac{1}{\left(q_{z}-q_{\mathrm{mf}}\right)^{2-\eta_{m}}} \\
S\left(q_{z}=q_{\mathrm{mf}}, q_{\perp} \simeq 0\right) \propto \frac{1}{q_{z}^{4-2 \eta_{m}}} .
\end{gathered}
$$

The exponent $\eta_{m}$ is a measure of the tail-to-peak intensity and is expected to increase with the interlayer distance due to the fact that the steric repulsion, though the compression modulus $\bar{B} \sim 1 / d_{f}^{3}$, is decreasing:

$$
\eta_{m}=\frac{q_{\mathrm{mf}}^{2}}{8 \pi \sqrt{\bar{B} \kappa_{c}}}
$$

In the work of Yang et al. [5] the reduction of the peak width with increasing fraction of polymer grafted to $\mathrm{C}_{12} \mathrm{E}_{5}$ membrane (at fixed membrane volume fraction) was associated with a decrease in the exponent $\eta_{1}$. In our case it is observed that $\eta_{1}$, determined from Eq. (19) is decreasing with the polymer length (at fixed polymer and membrane volume fractions). Note, however, that exact values of $\eta_{1}$ from peak shape analysis suffer from the limited $q$ resolution of SANS and that the main objective of this paper is the investigation of the membrane fluctuation dynamics by NSE spectroscopy.

\section{B. Neutron spin-echo results}

All NSE experiments were conducted at the FRJ2-NSE [24] in the cold neutron guide hall ELLA in Jülich. A wavelength band centered at $\lambda=0.8 \mathrm{~nm}$ with a width full width at half maximum (FWHM) of $10 \%$ has been used throughout. Due to the use of a 2D-area detector each angle setting of the scattering arm yielded up to five separate curves corresponding to different $q$ values. For each sample 4-5 different scattering angle settings covering the range $0.35 \leqslant q \leqslant 2.6 \mathrm{~nm}^{-1}$ were employed. The sample holders were oriented with respect to the neutron beam such that the rotation of the sample table together with the movement of the detection arm of the instrument assures that the scattering vector $\vec{q}$ is oriented

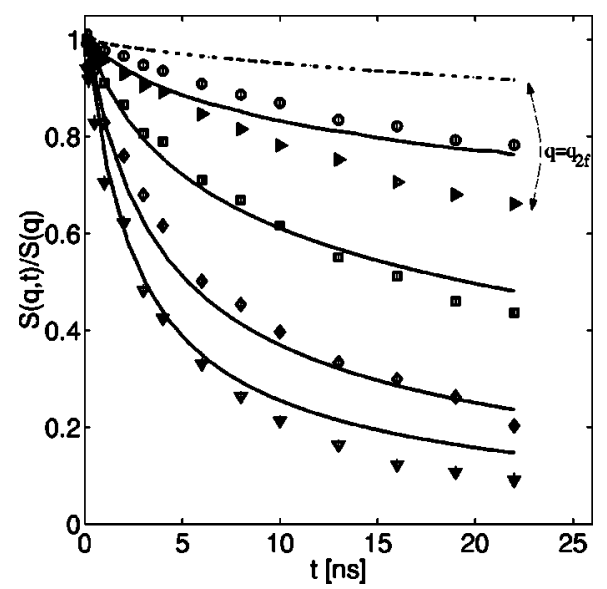

FIG. 7. Comparison of the NSE experimental data (symbols) for the $\mathrm{PEP}_{5}-\mathrm{PEO}_{5}$ system to the numerically evaluated $S(q, t) / S(q)$ (solid lines) based on the Caillé approach for a range of $q$ values $0.8-2.2 \mathrm{~nm}^{-1}$. The dashed line is the calculated curve and the experimental $(\triangleright)$ data, respectively, at $q=1 \mathrm{~nm}^{-1}$ — the secondorder pseudo-Bragg peak position.

always in the direction perpendicular to the lamellar planes (i.e., $\overrightarrow{\mathrm{q}} \equiv q_{z}$ ). This orientation assures the highest scattered intensity, around and beyond the peak position. Knowing the position of the first-order pseudo-Bragg peaks $\left(q_{z}=q_{1 \mathrm{f}}, q_{\perp}\right.$ $=0$ ), the dynamics is investigated along a line $\overrightarrow{\mathrm{q}} \| q_{z}$ at $q$ $\geqslant q_{1 \mathrm{f}}$, up to $q \simeq(4 \cdots 5) q_{1 \mathrm{f}}$, where the intensity did not yet dive too much into the incoherent background. The positions of the points in reciprocal space where the NSE experiments were performed are indicated by the circles in Fig. 4 along the $0^{\circ}$ cut line through the detector intensity map.

\section{Intermediate scale dynamics}

It is expected that the static correlations leading to the sharp correlation (pseudo-) peaks are also reflected in the membrane dynamics at $q \geqslant q_{1 \mathrm{f}}$. In order to better account for the influence of the structure factor on the dynamics, the dynamic version of the membrane stack structure factor as formulated by Caillé [10] was tested against the NSE experimental data. Fixing the parameters $d_{f}, \kappa$, and $\bar{B}$ already determined from the SANS analyses, the time-dependent expression [Eq. (12) inserted into Eq. (6)] is evaluated numerically for a range of $q$-values higher than $q=2 q_{1 \mathrm{f}}$ and compared to the experimental data. Because of the very demanding and time consuming computational task, the NSE data were not fitted, rather the remaining parameters $\bar{\eta}$ and (somewhat more technical) $\epsilon$ were estimated by observing the quality of the agreement with the data. This is illustrated in Fig. 7 for the $\mathrm{PEP}_{5}-\mathrm{PEO}_{5}$ system. It can be inferred that the agreement with the data is reasonable with the exception of the $q \simeq 1 \mathrm{~nm}^{-1}$ curve represented with dashed line. This $q$ value corresponds to the position of the second-order scattering peak, although barely visible in the SANS pattern. The observation indicates that the observed intensity at the peak position contains considerabel off-peak contributions due to the limited $q$ resolution of the NSE setup. Therefore, the slowing down at the peak is less pronounced than antici- 
pated. Howevere, a full resolution convolution was prevented by the excessively long computation times. Away from the peaks with their steep variation with respect to $q$, resolution effects are of minor importance. Assuming that the bending modulus is $\kappa=0.728 k_{B} T$ from the SANS evaluations, and $\Gamma_{q}=\kappa /(4 \eta) q^{3}$ the remaining undetermined parameters which could best reproduce the experimental curves (simultaneously, for all $q$ values) are: $\eta=1.7 \mathrm{cP}$ and $k_{\text {min }}$ $=1 \mathrm{~nm}^{-1}$. On the other hand viscosity of the solvent $\eta$ $=0.86 \mathrm{cP}$ at $26^{\circ} \mathrm{C}$ was evaluated from the average of the table values [24-26] for water and decane. The required viscosity is thus, about two times higher than expected for water + decane. It is anyhow expected that the presence of the polymer coils attached to the membranes will increase the dissipation (within the membrane or in the surrounding fluid) but this contribution can presently not be quantified on a theoretical basis.

\section{Local scale dynamics}

In the regime of high $q\left(q d_{f} \gg 1\right)$, it may be assumed that the influence of the intermembrane interactions on the dynamic structure factor are small, and the data can be described by the expression derived by Zilman and Granek [17] for a single-membrane patch. In the present experiment on an oriented lamellar phase the direction of the scattering wave-vector relative to the membrane planes is set to $\vec{q}$ $=\left(0,0, q_{z}\right)$. No angular averaging is necessary in contrast to the bicontinuous phase [18]. The extension of validity of the Zilman and Granek theoretical prediction to the lamellar phase is inspected by stretched exponential analysis and by full numerical calculations based on Eq. (A15). A first data analysis in terms of the exponents $\alpha$ and $\beta$ in $S(q, t) / S(q)$ $=\exp \left[-\left(\Gamma q^{\alpha}\right)^{\beta}\right]$, i.e., $\Gamma_{q}=\Gamma q^{\alpha}$, is compared with the predictions of the single-membrane approach [17]. In Fig. 8 NSE relaxation curves corresponding to the basic $\mathrm{C}_{10} \mathrm{E}_{4}$ system are shown. The data (symbols) were fitted with stretched exponential functions with $\Gamma_{q}$ and $\beta$ in Eq. (17) as fit parameters. The dotted and dashed lines correspond to $q \simeq q_{1 \mathrm{f}}$ and $q \simeq q_{2 f}$, respectively. The observed slowing down is more directly visible in the two minima of $\Gamma_{q}$ represented in Fig. 9(left). As it is observed also from the values of the exponent $\beta$ in Fig. 9 (right), no simple scaling behavior is found in the accessed $q$ range. The evolution of the relaxation rate with the scattering wave vector observed in Fig. 9 (left) indicates that to a large extent the dynamics is influenced by the structure which appears as subsequent minima of $\Gamma_{q}$ corresponding to the correlation peaks. Although the SANS measurement were not extended up to the position of the secondorder peak [see Fig. 3(a)], a slowing down in the relaxation corresponding to $q_{1 \mathrm{ff}}$ and also $q_{2 f}$ is present in the NSE results. Since for this pure $\mathrm{C}_{10} \mathrm{E}_{4}$ system the lamellar phase can only be obtained at higher surfactant concentrations, the first correlation (pseudo-Bragg) peak position is situated at quite large wave vectors $\left(q_{1 \mathrm{f}}=1.37 \mathrm{~nm}^{-1}\right)$. Reaching the asymptotic high- $q$ regime which was seen to occur at $q$ $>4 q_{1 \mathrm{f}}$ in the bicontinuous phase, would require (by analogy) $q>2 q_{1 \mathrm{f}} \simeq 2.74 \mathrm{~nm}^{-1}$, where the incoherent scattering becomes important. A decomposition of coherent and incoher-

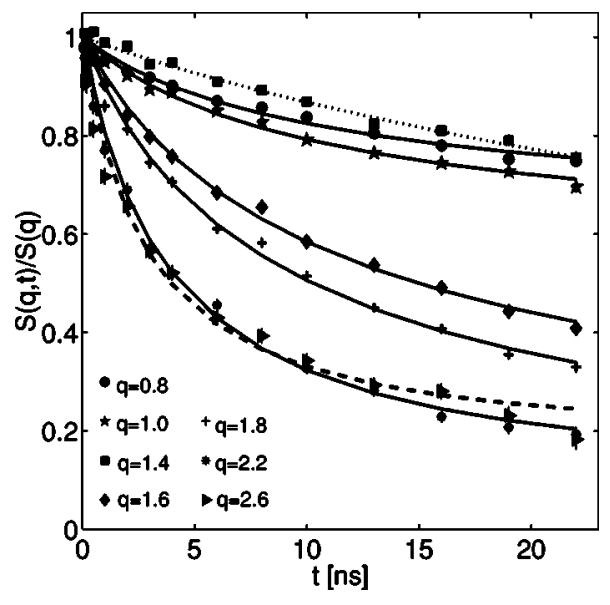

FIG. 8. NSE relaxation curves for the pure $\mathrm{C}_{10} \mathrm{E}_{4}$ system in lamellar phase (symbols) and fits of the data with stretched exponential function (lines). The dotted and dashed lines correspond approximately to the first- and second-order scattering peaks, respectively, at $q_{1 \mathrm{f}}=1.37 \mathrm{~nm}^{-1}$ and $q_{2 f}=2.74 \mathrm{~nm}^{-1}$.

ent scattering then becomes difficult or intractable and without further knowledge the interpretation of these data would become questionable. The values of $\beta$ found in the accessible $q$-range are-except for $q_{1 \mathrm{f}}$-in general less than 1, i.e., the relaxation is nonexponential. This may be explained by the mixing of various exponential relaxation modes with different rates $\omega(\overrightarrow{\mathrm{k}})$ contributing to the scattering function at the respective wave vector $\overrightarrow{\mathrm{q}}$.

A stretched exponential analysis performed for the systems with polymers yielded the results gathered in Fig. 10 for the relaxation rates $\Gamma_{q}$ and $\beta$ exponents. The NSE investigated $q$-range extends up to $0.22 \mathrm{~nm}^{-1}$. In terms of reduced wave vectors $q^{*}=q / q_{1 \mathrm{f}}$ the following values are reached: $q^{*}=4.2$ for $\mathrm{PEP}_{5}-\mathrm{PEO}_{5}, q^{*}=4$ for $\mathrm{PEP}_{2}-\mathrm{PEO}_{2}$, and $q^{*}=3.7$ for $\mathrm{PEP}_{1}-\mathrm{PEO}_{1}$, respectively. As it is observed from Fig. 10 a $q^{3}$ dependence of the relaxation rate and $\beta$ exponents approaching $2 / 3$ are found at $q \geqslant(3 \cdots 4) q_{1 \mathrm{f}}$. A slowing-down in the relaxation was also observed at $q$ $=q_{2 f}$, although the second-order peak is barely visible in the intensity maps presented in Fig. 3(b)-3(d). Due to the limited range of $q$ values around and below the first-order peaks, a minimum at $q=q_{1 \mathrm{f}}$ could only be guessed from the relaxation rate behavior in Fig. 10 (left). Overall, the results are in qualitative agreement-in terms of the scaling exponents $\alpha$ and $\beta$-with the approximative Zilman-Granek results [17] in the asymptotic regime. The same kind of agreement was obtained previously for bicontinuous phases of similar compositions [18,27].

For a complete analysis and the determination of the bending modulus $\kappa$, the full numerical procedure as described in Eq. (16), with the corresponding integration limits, was carried out. Since it is not clear what is the relevant correlation length for the lamellar phase, the parameter $\epsilon$ was also fitted (besides the bending modulus $\kappa_{0}$ ). For exemplification, for the system containing $\mathrm{PEP}_{5}-\mathrm{PEO}_{5}$ Fig. 11 presents experimental data (symbols) together with the fits based on Eq. (16). For the $\mathrm{PEP}_{5}-\mathrm{PEO}_{5}$ and $\mathrm{PEP}_{2}-\mathrm{PEO}_{2}$ sys- 

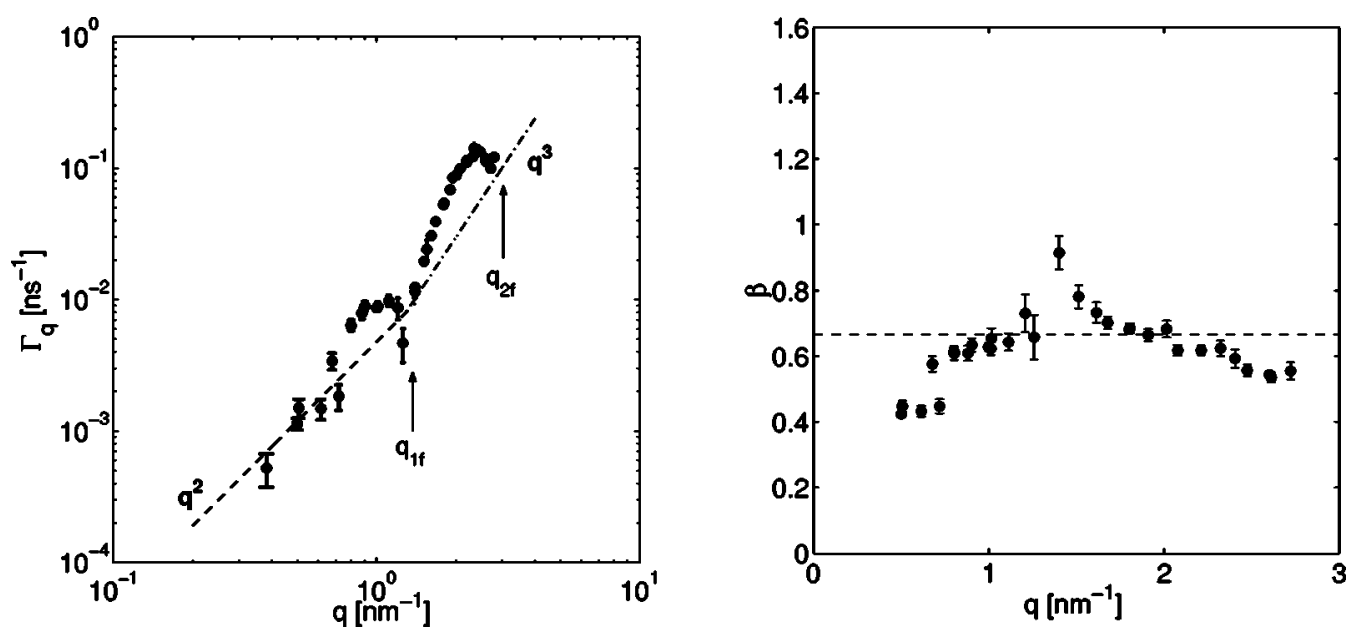

FIG. 9. Left: Relaxation rate $\Gamma_{q}(q)$ from the stretched exponential analysis based on Eq. (17) for the basic $\mathrm{C}_{10} \mathrm{E}_{4}$ system. Right: Corresponding $\beta(q)$ exponent; the dashed horizontal line indicates the $\beta=2 / 3$ level.

tems the obtained (bare) $\kappa_{0}$ values are presented in Fig. 12 versus the wave vector $q$ of the fitted relaxation curve. The integration limit scaling factor $\epsilon$ is also shown (insert). The meaningful observation from this results is that, for some high- $q$ range, again in the limits $q \geqslant(3 \cdots 4) q_{1 \mathrm{f}}$, the $\kappa$ values (and the factor $\epsilon$ ) apparently converge to a constant level. I.e., the model describes all data beyond that $q$-value consistently with one set of parameters. Average values of $\kappa_{q \geqslant 4 q_{1 f}}=1.98 k_{B} T \quad\left(\mathrm{PEP}_{5}-\mathrm{PEO}_{5}\right) \quad$ and $\quad \kappa_{q \geqslant 4 q_{1 f}}=1.72 k_{B} T$ $\left(\mathrm{PEP}_{2}-\mathrm{PEO}_{2}\right)$ were determined from the fits. Correspondingly, average values of $\epsilon=0.64$ and $\epsilon=0.61$ were found for the two systems. Due to the fact that the apparent values $\kappa(q)$ from the $\mathrm{PEP}_{1}-\mathrm{PEO}_{1}$ system are more scattered, (probably due to worse counting statistics of these data) the results were not displayed in the figure. Also, the available data points in the range $q \geqslant(3 \cdots 4) q_{1 \mathrm{f}}$ is very limited in this case. For the highest $q=2.2 \mathrm{~nm}^{-1}$ measurement at this system, the apparent (fitted) values are $\kappa=1.29 k_{B} T$ and $\epsilon$ $=0.6$.

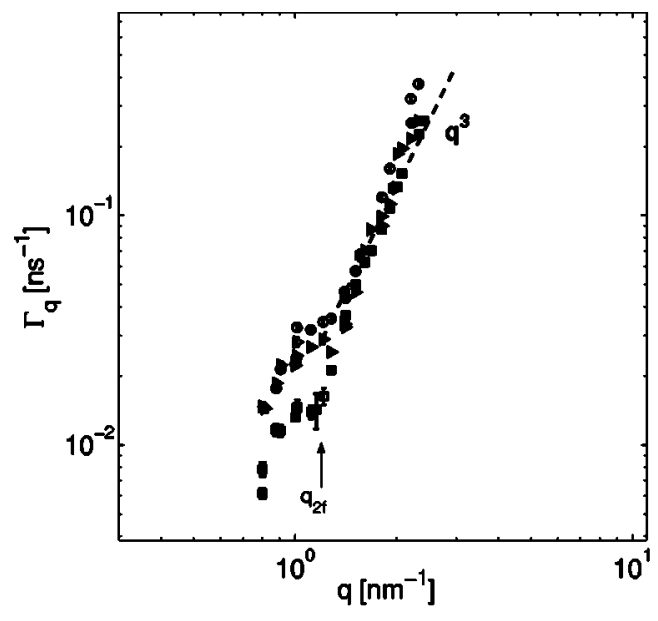

\section{DISCUSSION}

The examined systems provide a test of the theories describing the single layer (at high- $q$ ) or multilayer membrane systems (at low $q$ ). The underlying elastic theory was proposed by Landau, Peierls, and de Gennes [28,29] for smectic- $A$ liquid crystals.

A good description of the peak shape, with values of the physical parameters in agreement with previous results on similar systems $[5,30]$ was achieved by using the complete numerical evaluation of the structure factor in the Caille [10] formulation. The difference between the bending elasticity moduli of $\kappa \simeq 0.8 k_{B} T$ found by this procedure, and previous SANS results for the bicontinous phase [which yield $\kappa$ $\simeq 1.3 k_{B} T$ [8]] represents the degree of accuracy achieved. Sources for systematic errors are the approximations made and the significant instrumental resolution effects.

The compression modulus $\bar{B} \leqslant 10^{5} \mathrm{~Pa}$ corresponding to interlayer distances of $\sim 10 \mathrm{~nm}$ is comparable to the values in the range of $\bar{B} \simeq 6 \times 10^{6} \mathrm{~Pa}$ determined for SDS lamellar

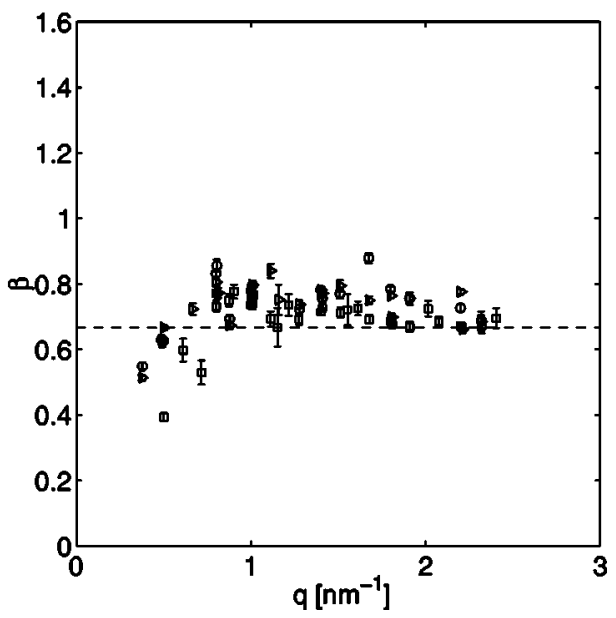

FIG. 10. Left: Relaxation rates $\Gamma_{q}(q)$ and exponents $\beta(q)$ from a stretched exponential analysis corresponding to: $\mathrm{PEP}_{1}$ - $\mathrm{PEO}_{1}(\mathrm{O})$, $\mathrm{PEP}_{2}-\mathrm{PEO}_{2}(\triangleright)$, and $\mathrm{PEP}_{5}-\mathrm{PEO}_{5}(\square)$. Right: Corresponding $\beta(q)$ exponents. 


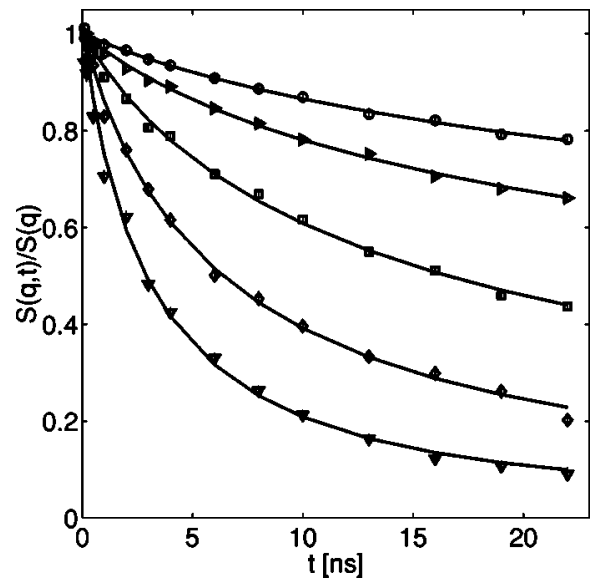

FIG. 11. NSE relaxation curves for oriented lamellar phase with $\mathrm{PEP}_{5}-\mathrm{PEO}_{5}$ corresponding, from top to bottom, to $q$ $=(0.5,0.8,1.0,1.4,1.8,2.2) \mathrm{nm}^{-1}$. The solid lines are individual fits of the data with the full numerical treatment using Eq. (16).

systems for intermembrane distances of $\sim 6 \mathrm{~nm}[2,30]$. However, not much can be argued on the membrane interaction from the apparent values of $\bar{B}$. The dependence $\bar{B}$ $\sim 1 / d_{f}^{3}$ would render the opposite (and anyhow, larger) variation between the systems than the apparent $\bar{B}$ determined by fits (see Table III). The apparent values of the parameters can be influenced by collective (surfactant+polymer) effects. For instance, the polymers extending in the solvent may contribute to the steric membrane repulsion. The range of this effective contribution is expected to increase with polymer length, which becomes as large as the interlayer distance for the longest polymer $\left(\mathrm{PEP}_{5}-\mathrm{PEO}_{5}\right)$. Studies on the lamellar phases of nonionic surfactant membranes doped with small amounts of anionic surfactants (e.g., SDS), [3,31] revealed an increase in the interlayer spacing $d$ upon layer de-

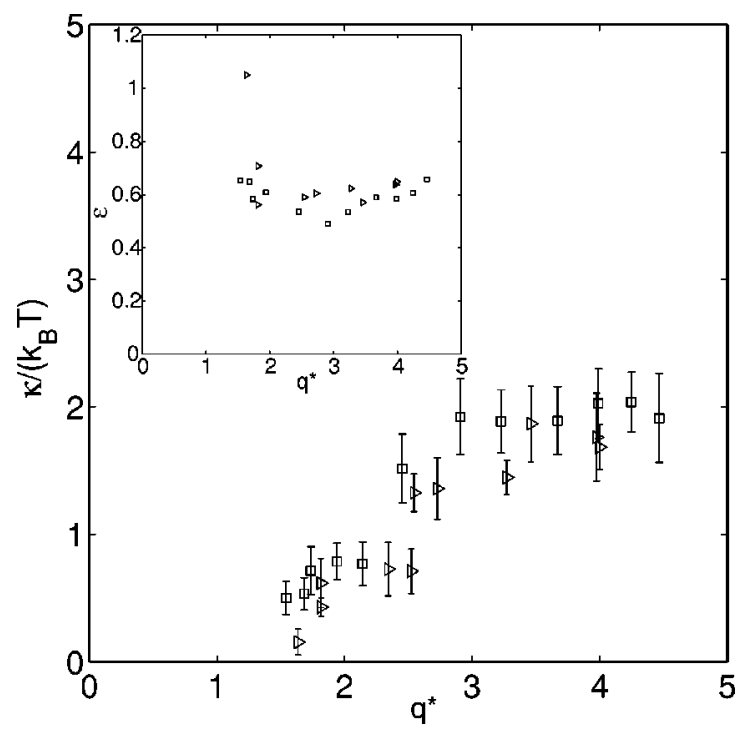

FIG. 12. The bending modulus $\kappa\left(q^{*}\right)$ determined from fits with Eq. (16), versus the reduced wave vector $q^{*}=q / q_{1 \mathrm{f}}$ for $\mathrm{PEP}_{2}-\mathrm{PEO}_{2}$ $(\triangleright)$ and $\mathrm{PEP}_{5}-\mathrm{PEO}_{5}(\square)$. The insert shows the corresponding scaling factors $\epsilon$ applied to the integration limits. charging (i.e., increasing the screening ion concentration in solution). This was explained by a "crumpling" of the surfactant layer due to decreasing electrostatic repulsion. By analogy, the observation of increasing $d_{f}$ for longer polymers (i.e., lower polymer surface density) could be attributed to a crumpling of the layer. It can be shown $[2,32]$ that, if the Helfrich undulation interaction is dominant and provided that the system's Hamiltonian is described correctly by Eq. (1), the first-order exponent $\eta_{1}$ follows a simple geometrical expression

$$
\eta_{1}=\frac{4}{3}\left(1-\frac{a}{d_{f}}\right)^{2},
$$

where $a$ is the surfactant layer thickness. This picture was confirmed in the work of Safinya [2] in synchrotron X-ray studies on SDS lamellar phases along a dilution line. At the distances of $\sim 10 \mathrm{~nm}$ found for our systems, it is expected that the Helfrich steric repulsion (per unit area) $V_{s}$ $\simeq 0.32 k_{B} T^{2} /\left(\kappa d_{f}^{2}\right)$ [1] is about one order of magnitude higher than the van der Waals attraction $V_{v d W} \simeq$ $-0.75 k_{B} T a^{2} / d_{f}^{4}$ [2]. Amphiphilic block-copolymer anchored or grafted to the surfactant layers enhances the repulsive interlamellar forces as revealed by an enhancement of the Bragg singularities in the scattering intensity [5,33]. Castro-Roman et al. [6] have demonstrated in a series of SANS studies of lamellar phases with grafted triblockcopolymers that the polymers contribute by an osmotic repulsion to the effective steric interaction between membranes. It was suggested that this effective steric repulsion can be viewed as a long-range renormalized Helfrich interaction, where the layer thickness, $a_{\mathrm{eff}}=a+R_{g}^{\mathrm{PEP}}+R_{g}^{\mathrm{PEO}}$, is the effective thickness containing contributions from the polymer gyration radius $R_{g}$. This effective thickness can be calculated in our case from the $\mathrm{C}_{10} \mathrm{E}_{4}$ layer thickness of $a$ $\simeq 1.2 \mathrm{~nm}$ [8] and $R_{g} \simeq R_{e} / \sqrt{6}$, where the polymers end-toend distances are listed in Table II. The values of $a_{\text {eff }}$ $=(3.98,4.96,6.89)-$ in the order of increasing polymer size-thus estimated, can be compared to $a$ $=(7.82,8.26,8.58)$ calculated from Eq. (20) with $d_{f}$ and $\eta_{1}$ determined from the fits. As it can be observed, the values are of comparable magnitudes, but the slope of the variation, versus polymer size, is different. A more precise verification would require studies on a series of lamellar phases along a dilution line, keeping the polymer type and density fixed. This is not possible for this nonionic surfactant systems which exhibits lamellar phases only at higher concentrations and-in the presence of membrane-anchored blockcopolymers-additional narrow lamellar islands at lower concentrations.

The examined $q$ range in the NSE investigations, sometimes extending to $q \geqslant 4 q_{1 \mathrm{f}}$ allows for a test of the singlemembrane dynamics description of Zilman and Granek. The results for the NSE data at the highest scattering wave numbers $q$ confirm the picture of single membranes which relax through undulation mode (membrane Zimm dynamics). The determined effective bending modulus $\kappa \simeq 1.9 k_{B} T$ (for $12 \%$ polymer in surfactant) is not too different from values of $1.3 k_{B} T$ determined for the bicontinuous phases [8,18] (for 
$10 \% \mathrm{PEP}_{10}-\mathrm{PEO}_{10}$ in surfactant). It should be mentioned that since the dynamics also dependends on the dissipation $(\propto \eta)$, as well as on the restoring forces $(\propto \kappa, \bar{B})$, possible effects on the viscosity, i.e., the membrane internal viscosity [34], or an increased friction with the solvent due to the anchored polymers may influence the results. In the present analysis only bulk fluid viscosities were assumed.

\section{CONCLUSION}

The lamellar phase of a nonionic surfactant with water and oil in equal proportions as studied here allows for investigation of monolayer membranes properties. The noncharged membranes are free of Coulombic interaction, i.e., exhibit mainly steric interaction, and since they are surfactant monolayers, intramembrane dissipation should be small — which may be more important in the case of bilayers. However, the lamellar islands in the phase diagrams are of limited size, such that equal membrane distance could only be maintained for the series of polymer-containing systems, whereas the pure surfactant systems was only available with a smaller $d$ spacing. Oriented samples were prepared for all systems and investigated by SANS. Here it should be kept in mind that the primary purpose of the present investigation was the study of fluctuation dynamics by NSE for which the SANS experiments serve as background information on $S(q, t=0)$. However, even with the-compared to x-ray/ synchrotron radiation diffraction-poor $q$ resolution of the SANS an estimation of the relevant parameters of the membrane stack could be obtained. The existent theoretical treatments, based on the phenomenological elastic interface model, succeed in reproducing the typical features of the SANS and the NSE experimental data. We attempted to show which are the approaches or conditions of using them in order to obtain quantitative estimates of the membrane elastic properties, consistent with physical arguments [35] and previous neutron scattering results [8]. It was demonstrated that the treatment of the membrane stack in the formulation of Caillé compare well with the experimental data around the first pseudo-Bragg peak. However, large effects of the instrumental resolution may blurr details and hide higher-order pseudo-Bragg peaks. Even quantitative measures of the bending modulus $\kappa \simeq 0.8 k_{B} T$ and compression modulus $\bar{B} \simeq 0.1 k_{B} T / \mathrm{nm}^{3}$ may be obtained. It is recommendable to determine the energetic and structural parameters like $\kappa, \bar{B}$ or $d_{f}$ from diffraction (SANS, x-ray) measurements. The size of $\kappa$ reasonably agrees with earlier findings in previous studies on the bicontinuous [18] or droplet phases [36]. In the NSE investigations a qualitative description (see Fig. 7) of the relaxation curves $S(q, t) / S(q)$ could be achieved by the introduction of the simplified (asymptotic) mode dispersion $\omega\left(k_{\perp}\right) \propto k_{\perp}^{3}$ into the expression for $S(q)$, Eqs. (6), (7), and (12). It is remarkable that despite the fact that the mode wave vector $\overrightarrow{\mathrm{k}}_{\perp}$ in this approximation is perpendicular to the experimental $\vec{q}$ vector, relaxations due to membrane fluctuation with qualitative correct behavior are predicted. This illustrates the strong mode mixing to be expected in the experimental signal. Also slowing-down at the peak positions is predicted and clearly observed. However, here resolution effects reduce the size of the observed effect. In general the low $q$ data $\left(q<3-4 q_{1 f}\right)$ should suffer from the fact that the used mode dispersion is only valid asymptotically. In the asymptotic high- $q$ regime a quantitative extraction of the membrane bending modulus $\kappa$ seems to be possible. The value depends on the exact choice of the integration cutoff at low mode wave numbers. Fitting suggests that the effective mode spectrum extends to $\simeq 0.6 \pi / d_{f}$ which is consistently found in the asymptotic regime. A clear distinction between the $\kappa$ values for the different polymers was not observed.

In principle the measured dynamics depends on the ratio of the bending modulus $\kappa$ and the friction. As long as it is assumed that the friction is only due to the average bulk viscosity $\bar{\eta}$ of the surrounding fluids (water and oil) $\kappa$ can be determined from the asymptotic (high- $q$ ) NSE data. By this procedure values of $\kappa \simeq 1.8 k_{B} T$ are obtained, which are higher than the-not very accurate-estimate of $\kappa$ $\simeq 0.8 k_{B} T$ from the analysis of the peak shape in $S(q)$ and the values of $\kappa \simeq 1.3-1.5 k_{B} T$ for the bicontinuous phase $[8,18]$. This suggests an underestimation of the dissipation. Because of these uncertainties it is difficult at this point to discriminate between the effects of the polymer presence on either the membrane rigidity or the friction. Further investigations on more dilute systems, alternatively using $\mathrm{x}$ ray for a better $q$ resolution, combined with NSE measurements would help answering some of the questions posed here.

\section{APPENDIX: STRUCTURE FACTOR OF A LAMELLAR MEMBRANE SYSTEM}

Starting from Eq. (3) in Sec. II the membrane stack structure factor can be written as

$$
\begin{aligned}
\begin{aligned}
S(q) & =\sum_{n} \int d^{2} \vec{\rho} e^{i q_{z} n d_{f}} e^{\overrightarrow{q_{\perp}} \vec{\rho}_{G}} G\left(\vec{r}, q_{z}\right) \\
& =2 \pi \sum_{n} e^{i q_{z}\left(n d_{f}\right)} \underbrace{\int d \rho \rho J_{0}\left(q_{\perp} \rho\right) G\left(\vec{r}, q_{z}\right)}_{\left.I_{\vec{\rho}}\right)},
\end{aligned} \\
\text { where } \vec{r}=\left(\vec{\rho}, n d_{f}\right) \text { and }
\end{aligned}
$$

$$
G\left(\vec{r}, q_{z}\right)=e^{-k_{B} T /(2 \pi)^{3} q_{z}^{2}} \overbrace{\int d^{3} \overrightarrow{\mathrm{k}} \frac{\left(1-e^{-i \overrightarrow{\mathrm{k}} \mathrm{r}}\right)}{\bar{B} k_{z}^{2}+\kappa_{c} k_{\perp}^{4}}}^{I_{\vec{k}}} .
$$

Separating in the components perpendicular and parallel to the lamellar plane, the integral $I_{\overrightarrow{\mathrm{k}}}$ becomes

$$
\begin{aligned}
I_{\overrightarrow{\mathrm{k}}} & =2 \pi \int_{-\infty}^{\infty} d k_{z} \int_{0}^{\infty} d k_{\perp} k_{\perp} \frac{1-e^{i k_{z}\left(n d_{f}\right)} J_{0}\left(k_{\perp} \rho\right)}{\bar{B} k_{z}^{2}+\kappa_{c} k_{\perp}^{4}} \\
& =\frac{4 \pi}{\bar{B}} \int_{0}^{\infty} d k_{\perp} \underbrace{k_{\perp} \int_{0}^{\infty} d k_{z} \frac{1-\cos \left(k_{z} n d_{f}\right) J_{0}\left(k_{\perp} \rho\right)}{k_{z}^{2}+\alpha k_{\perp}^{4}}}_{I_{k z}},
\end{aligned}
$$


where we have defined $\alpha=\kappa_{c} / \bar{B}$. Following Ref. [37] the integral over $k_{z}$ in the above equation can be further carried out:

$$
I_{k z}=\underbrace{\int_{0}^{\infty} d k_{z} \frac{k_{\perp}}{k_{z}^{2}+\alpha k_{\perp}^{4}}}_{I_{k z 1}}-\underbrace{k_{\perp} \int_{0}^{\infty} d k_{z} \frac{J_{0}\left(k_{\perp} \rho\right) \cos \left(k_{z} n d_{f}\right)}{k_{z}^{2}+\alpha k_{\perp}^{4}}}_{I_{k z 2}},
$$

$$
I_{k z 1}=\frac{\pi}{2} \frac{1}{\sqrt{\alpha} k_{\perp}}
$$

The integral $I_{k z 2}$ is solved by contour integration with the pole $+i \sqrt{\alpha} k_{\perp}^{2}$ (in the upper-half-plane), to yield

$$
I_{k z 2}=\frac{\pi}{2} \frac{1}{\sqrt{\alpha} k_{\perp}} e^{-\sqrt{\alpha} k_{\perp}^{2}\left(n d_{f}\right)} J_{0}\left(k_{\perp} \rho\right) .
$$

Inserting the series expansion of the Bessel function $J_{0}(x)$ $=\Sigma_{n=0}(-1)^{n} x^{2 n} /\left(2^{2 n} n !^{2}\right)$ into Eq. (A3) yields

$$
\begin{aligned}
& I_{\overrightarrow{\mathrm{k}}}=\frac{4 \pi^{2}}{2 \sqrt{\alpha} \bar{B}} \underbrace{\int_{0}^{k_{\max }} \frac{d k_{\perp}}{k_{\perp}}\left(1-e^{-\sqrt{\alpha} k_{\perp}^{2}\left(n d_{f}\right)}\right)}_{I_{k 1}} \\
& -\underbrace{\int_{0}^{k_{\max }} \frac{d k_{\perp}}{k_{\perp}} \sum_{n=1} \frac{(-1)^{n}\left(k_{\perp} \rho\right)^{2 n}}{2^{2 n} n !^{2}} e^{-\sqrt{\alpha} k_{\perp}^{2}\left(n d_{f}\right)}}_{I_{k 2}} .
\end{aligned}
$$

The first integration yields

$$
\begin{aligned}
I_{k 1}= & \frac{2 \pi^{2}}{\sqrt{\alpha} \bar{B}}\left[\ln \left(k_{\max }\right)+\frac{1}{2} E_{1}\left(\sqrt{\alpha} k_{\max }^{2} n d_{f}\right)+\frac{1}{2} \gamma\right. \\
& \left.+\frac{1}{2} \ln \left(\sqrt{\alpha} n d_{f}\right)\right]
\end{aligned}
$$

where $k_{\max }$ is the small wavelength cutoff of the order of $\pi / a, E_{1}(x)=\int_{\infty}^{x} e^{t} / t d t$ is the exponential integral, and $\gamma$ $=0.5772 \ldots$, is the Euler constant. With a variable substitution $u=k_{\perp} \rho$, the second integral of Eq. (A7) can be expanded

$$
\begin{aligned}
I_{k 2} & =\frac{2 \pi^{2}}{\sqrt{\alpha} \bar{B}} \int_{0}^{u_{\max } / \rho} d u \sum_{n=1} \frac{(-1)^{n} u^{2 n-1}}{2^{2 n} n !^{2}} e^{-\sqrt{\alpha}\left(n d_{f}\right) u^{2} / \rho^{2}} \\
& =\frac{2 \pi^{2}}{\sqrt{\alpha} \bar{B}} \sum_{n=1} \frac{(-1)^{n}}{2^{2 n} n !^{2}} \int_{0}^{u_{\max } / \rho} d u u^{2 n-1} e^{-w u^{2}},
\end{aligned}
$$

where $k_{\max }=u_{\max } / \rho$ and $w=\sqrt{\alpha} z / \rho^{2}$, and $z=n d_{f}$. It can be shown that, in general $\int_{0}^{\infty} d u u^{2 n-1} e^{-w u^{2}}=\frac{1}{2}(n-1) ! w^{-n}=\frac{1}{2}(n-1) !(4 w)^{-n} 2^{2 n}$

and

$$
E_{1}(x)=\gamma+\ln (x)+\sum_{n=1}^{\infty} \frac{(-1)^{n} x^{n}}{n n !} .
$$

Using the above expression and letting $k_{\max } \rightarrow \infty$ in Eq. (A9) only, Eq. (A9) becomes

$$
\begin{aligned}
I_{k 2} & =\frac{\pi^{2}}{\sqrt{\alpha} \bar{B}} \sum_{n=1} \frac{(-1)^{n}}{n n !}\left(\frac{1}{4 w}\right)^{n} \\
& =\frac{\pi^{2}}{\sqrt{\alpha} \bar{B}}\left[E_{1}(1 / 4 w)-\gamma-\ln (1 / 4 w)\right] .
\end{aligned}
$$

Returning to the integral over $k$, Eq. (A7) becomes

$$
\begin{aligned}
I_{\overrightarrow{\mathrm{k}}}= & \frac{\pi^{2}}{\sqrt{\alpha} \bar{B}}\left[2 \gamma+\ln \left(\frac{k_{\max }^{2} \rho^{2}}{4}\right)+E_{1}\left(k_{\max }^{2} \sqrt{\alpha} n d_{f}\right)\right. \\
& \left.+E_{1}\left(\frac{\rho^{2}}{4 \sqrt{\alpha} n d_{f}}\right)\right] .
\end{aligned}
$$

Substituting Eq. (A13) in Eq. (A2) and further, Eq. (A2) in Eq. (A1) we obtain finally

$$
\begin{aligned}
I_{\dot{\rho}}^{*}= & e^{-\mu / 2\left[2 \gamma+E_{1}\left(k_{\max }^{2} n d_{f} \sqrt{\alpha}\right)\right]} \frac{1}{q_{\perp}^{2}}\left(\frac{k_{\max }}{2 q_{\perp}}\right)^{-\mu} \\
& \times \int_{0}^{\infty} d x J_{0}(x) x^{1-\mu} e^{-\mu / 2 E_{1}\left[x^{2} /\left(4 \sqrt{\alpha} q_{\perp}^{2} n d_{f}\right)\right]}
\end{aligned}
$$

with $x=q_{\perp} \rho$ and $\mu=q_{z}^{2} /(4 \pi) k_{B} T /(\bar{B} \sqrt{\alpha})$. We thus came to the form of Eq. (A1) which was used in the fitting procedure

$$
\begin{aligned}
S(q)= & 2 \pi I_{\vec{\rho}}\left(n=0 ; q_{z}, q_{\perp}\right) \\
& +4 \pi \sum_{n=1}^{n=n_{\max }} \cos \left(q_{z} n d_{f}\right) I_{\vec{\rho}}\left(n ; q_{z}, q_{\perp}\right) .
\end{aligned}
$$

The Caille theory is considering an infinite number of lamellae in the stack. The finite size effect was accounted for by multiplying the quantity under the sum with a Gaussian having a width which covers a few hundreds nanometers along the normal to the lamellar planes. The other reason for this is to obtain convergence with a not too large number of layer in the summation and to avoid a (near) singularity in the resulting peak form. (The latter poses difficulties to subsequent resolution treatment). The summation is then carried out up to an $n_{\max }$ sufficiently large to assure the convergence of the sum. This limitation has the effect of rounding off the divergence (Bragg singularity) at the peak position $q=q_{m}$, however, to a much lower extent than the instrumental resolution. 
[1] W. Helfrich, Z. Naturforsch. A 33A, 305 (1978).

[2] C.R. Safinya, D. Roux, G.S. Smith, S.K. Sinha, P. Dimon, N.A. Clark, and A.M. Bellocq, Phys. Rev. Lett. 57, 2718 (1986).

[3] H. von Berlepsch and R. de Vries, Eur. Phys. J. E 1, 141 (2000).

[4] M.R. Kuzma, W. Wedler, A. Saupe, S. Shin, and Satyendra Kumar, Phys. Rev. Lett. 68, 3436 (1992).

[5] Y. Yang, R. Prudhomme, K.M. McGrath, P. Richetti, and C.M. Marques, Phys. Rev. Lett. 80, 2729 (1998).

[6] F. Castro-Roman, G. Porte, and C. Ligoure, Phys. Rev. Lett. 82, 109 (1999).

[7] B. Jakobs, T. Sottmann, R. Strey, J. Allgaier, L. Willner, and D. Richter, Langmuir 15, 6707 (1999).

[8] H. Endo, M. Mihailescu, M. Monkenbusch, J. Allgaier, G. Gompper, D. Richter, B. Jakobs, T. Sottmann, R. Strey, and I. Grillo, J. Chem. Phys. 115, 580 (2001).

[9] P.G. de Gennes, J. Phys. C 4, 65 (1969).

[10] M.A. Caillé, C. R. Seances Acad. Sci., Ser. B 274, 1733 (1972).

[11] J.S. Pedersen, D. Posselt, and K. Mortensen, J. Appl. Crystallogr. 23, 321 (1990).

[12] F. Brochard and J.F. Lennon, J. Phys. (Paris) 11, 1035 (1975).

[13] F. Nallet, D. Roux, and J. Prost, J. Phys. (France) 50, 3147 (1989).

[14] S. Ramaswamy, J. Prost, W. Cai, and T.C. Lubensky, Europhys. Lett. 23, 271, (1993).

[15] T. Schilling, O. Theissen, and G. Gompper, Eur. Phys. J. E 4, 103 (2001).

[16] R. Messager, P. Bassereau, and G. Porte, J. Phys. (Paris) 51, 1329 (1990).

[17] A.G. Zilman and R. Granek, Phys. Rev. Lett. 7, 4788 (1996).

[18] M. Mihailescu, M. Monkenbusch, H. Endo, G. Gompper, J. Stellbrink, D. Richter, B. Jakobs, T. Sottmann, and B. Farago, J. Chem. Phys. 115, 9563 (2001).

[19] S. Burauer, T. Sachert, T. Sottmann, and R. Strey, Phys. Chem. Chem. Phys. 1, 4299 (1999).

[20] J. Allgaier, A. Poppe, L. Willner, and D. Richter, Macromolecules 30, 1582 (1997).

[21] K.-D. Göcking, Ph.D. thesis, Westfälische Wilhelms Universität, Münster, Germany, 1997.
[22] C. Hiergeist and R. Lipowsky, J. Phys. II 6, 1465 (1996).

[23] G. Gompper, H. Endo, M. Mihailescu, J. Allgaier, M. Monkenbusch, D. Richter, B. Jakobs, and T. Sottmann, Europhys. Lett. 56, 683 (2001).

[24] M. Monkenbusch, R. Schaetzler, and D. Richter, Nucl. Instrum. Methods Phys. Res. A 399, 301 (1997).

[25] Handbook of Chemistry and Physics, 57th ed., edited by R.C. Weast (CRC Press, Cleveland, Ohio,1976).

[26] R.F. Berg and M.R. Moldover, J. Chem. Phys. 6 , 3687 (1987); D. Richter, B. Jakobs, and T. Sottmann, Europhys. Lett. (to be published).

[27] E. Freyssingeas, D. Roux, and F. Nallet, J. Phys. II 7, 913 (1997).

[28] L.D. Landau, in Collected Papers of L. S. Landau, edited by D. Ter Haar (Intern. Pub. Distr. Inc., New York, 1965); R.E. Peierls, Helv. Phys. Acta 7, 81 (1934).

[29] P.G. de Gennes, The Physics of Liquid Crystals (Clarendon, Oxford, 1974).

[30] K.D. Goecking and M. Monkenbusch, Europhys. Lett. 43, 135 (1998).

[31] R. Schomäcker and R. Strey, J. Phys. Chem. 98, 3908 (1994).

[32] D. Roux and C.R. Safinya, J. Phys. (France) 49, 07 (1988).

[33] H.E. Warriner, S.H.J. Idziak, N.L. Slack, P. Davidson, and C.R. Safinya, Science 271, 69 (1996).

[34] V. Lisy and B. Brutovsky, Phys. Rev. E 61, 4045 (2000).

[35] G. Gompper and G. Schick, in Phase Transitions and Critical Phenomena, edited by C. Domb and J. Lebowitz (Academic, London, 1994), Vol. 16.

[36] M. Mihailescu et al. (to be published).

[37] J. Als-Nielsen, J.D. Litster, R.J. Birgeneau, M. Kaplan, C.R. Safinya, A. Lindegaard-Andersen, and S. Mathiesen, Phys. Rev. B 22, 312 (1980).

[38] Note that the experimentally obtained phase diagram is displayed as a function of added surfactant mass fraction $\gamma$, since this value is well defined and directly obtained from the experimental procedure. The physically more relevant surfactant volume fraction (see Table I) is only derived using standard densities and taking into account the finite solubilities of $\mathrm{C}_{10} \mathrm{E}_{4}$ in the water respectively oil phase.

[39] Using this procedure, however, the resolution convolution was performed approximately taking into account values on the cut line only. 